\title{
Electrical and thermal efficiency of air-based photovoltaic thermal (PVT) systems: an overview
}

\author{
Nurul Shahirah Binti Rukman', Ahmad Fudholi², Ivan Taslim ${ }^{3}$, Merita Ayu Indrianti, \\ Intan Noviantari Manyoe ${ }^{5}$, Uce Lestari ${ }^{6}$, Kamaruzzaman Sopian ${ }^{7}$ \\ ${ }^{1,2,7}$ Solar Energy Research Institute, Universiti Kebangsaan Malaysia, Malaysia \\ ${ }^{3,4}$ Universitas Muhammadiyah Gorontalo, Indonesia \\ ${ }^{5}$ Universitas Negeri Gorontalo, Indonesia \\ ${ }^{6}$ Pharmaceutical Study Program, Faculty of Science and Technology, University of Jambi, Indonesia
}

\section{Article Info \\ Article history: \\ Received Sep 16, 2018 \\ Revised Nov 17, 2018 \\ Accepted Feb 28, 2019}

\section{Keywords:}

Efficiency

Energy analysis

Exergy analysis

Photovoltaic

Solar energy

\begin{abstract}
The development of photovoltaic thermal (PVT) system is a very promising area of research. PVT systems using in various applications, such as solar drying, solar cooling, water heating, desalination, and pool heating. With the recognition of the potentials and contributions of PV system, considerable research has been conducted to attain the most advancement which may produce reliable and sustainable PVT system. The cooling system's design refers to the absorber design which mostly focuses on water and air-based PVT systems. An air-based system has been developed through different absorber configurations, air flow modes and single- or double-pass design. Hence, a summarization on various research and development of air-based PVT system will be presented.
\end{abstract}

Copyright () 2019 Institute of Advanced Engineering and Science. All rights reserved.

\section{Corresponding Author:}

Ahmad Fudholi,

Solar Energy Research Institute, Universiti Kebangsaan Malaysia, 43600 Bangi Selangor, Malaysia. Email: a.fudholi@ukm.edu.my

\section{INTRODUCTION}

Solar energy is one of the sustainable energy which promises clean energy production. Regarding to limited conventional fuels, the implementation of solar technology is going into great advancement as one used to produce electrical energy using photovoltaic (PV) solar system. Furthermore, thermal energy can be generated which by utilizing working fluids in an integrated cooling system of PV; known as photovoltaic thermal (PVT) system. Regarding to the principle on how it works, electrical energy will be generated from a direct conversion of solar energy. The concept of PVT system in producing both electrical and thermal energy simultaneously had been studied in details. As heat from the sunlight or solar energy had been absorbed, the heat will be extracted by the air that flow in the cooling system. Focusing on air deployment as the coolant or working fluid, air-based PVT has been widely used for domestic application such as drying of agricultural products. Though, the study of PVT still experiencing great improvements in achieving great efficiency of energy production [1-13].

Energy and exergy analysis for PVT systems were studied base on theoretical and experimental study. Sarhaddi et al. [14], [15] performed a detailed energy and exergy analysis of an air-based PVT system to calculate the thermal and electrical parameters, exergy components and exergy efficiency of a typical airbased PVT system. The analysis showed that increasing inlet air velocity or solar radiation intensity, increased the exergy efficiency initially and then decreased after attaining a certain maximum inlet air velocity or solar radiation intensity. It was also reported that increasing wind speed increases the exergy 
efficiency. They also reported that the working fluid to have a significant effect on the exergy efficiency and that the exergy efficiency can be increased if an incompressible fluid (water) is used in PVT system. In this review, describe types of air-based PVT systems is presented. Also, electrical and thermal efficiency of airbased PVT systems base on energy and exergy analysis is presented.

\section{TYPES OF AIR-BASED PVT SYSTEMS}

PVT system which utilized air as the coolants in extracting heat from the PV panel had been employed to produced hot air aside the generation of electricity. An air-based PVT system consists of a PV panel, insulation and a frame as well as one or more glass cover or a transparent material placed over the absorbing plate (Figure 1) with air flowing around it. Based on air flow pattern, air-based PVT systems can be categorised into five types are: (i) conventional air-based PVT systems or as known back-pass air-based PVT system (Figure 2a), (ii) Double-pass with inlet air from channel above a PV panel (Figure 2b), (iii) single-pass parallel PVT system (Figure 2c), (iv) Single-pass with a channel above a PV panel (Figure 2d), and (v) double-pass with inlet air from channel above a PV panel (Figure 2e).

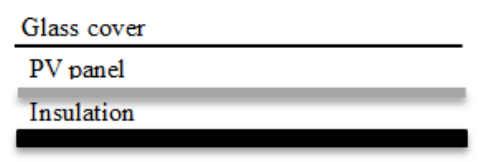

Figure 1. Configuration of air-based PVT system
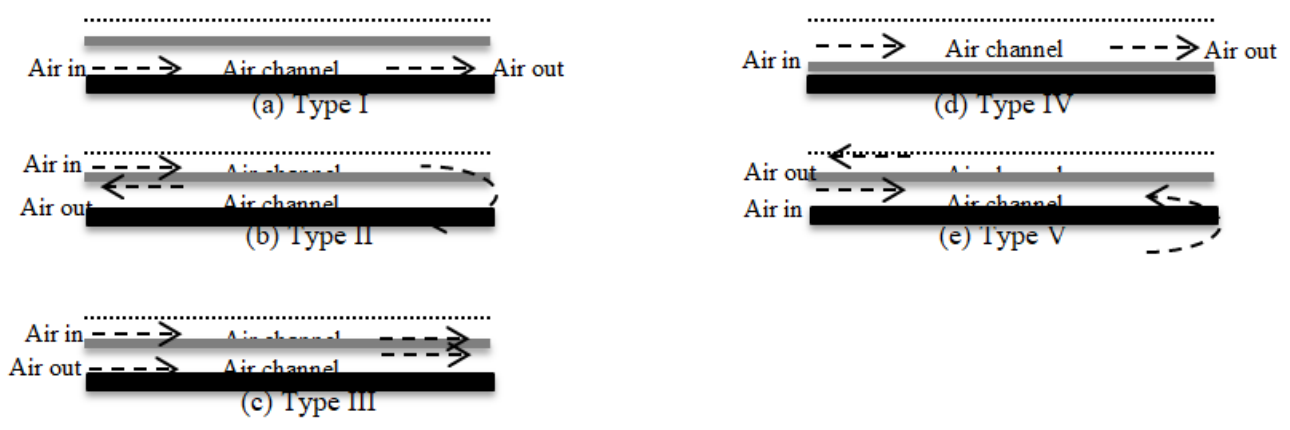

Figure 2. Sectional of air-based PVT systems [16] [17]

\section{STUDIES CONDUCTED ON AIR-BASED PVT SYSTEMS}

In PVT air-based, the air acts to cool down the panel. Several studies have been conducted during these recent years on the air hybrid collectors [18]. Early in 1996, a performance comparison on single and double pass PVT air collector had been conducted by Sopian et al. [19] which by then they concluded that the double pass-type PVT air collector portrayed better performance regarding the cooling of a solar cell.

One study focused by Hegazy [16] on such collectors and they had been evaluated through numerical modelling. Involved various designs of PVT air collectors such as: (i) air channel above PV collector, (ii) air channel below PV collector, (iii) single pass PV collector and (iv) double pass PV collector.

In 2006, Naveed et al. [20] experimentally investigated PVT grooved transpired plate performance. A range of $6 \mathrm{~K}$ of decreasing of module temperature had been observed. It resulted simple payback period of eight years, economically.

By using parameter or restriction of experiment carried out under no wind conditions and laminar flow, Arulanandam et al. [21] had conducted mathematical study of PVT. He numerically simulated a quarter of hole pitch and correlated numerical results in order to estimate Nusselt number as a function of suction velocity, hole diameter, plate thickness, and porosity.

Other numerical study also had been pursued by Li and Karava [22]. Heat transfer and air flow between PV panel and trapezoidal grooved transpired plate had been focused in this research. Nusselt number had been correlated by including length and height of groove, slope of trapezoidal corrugation, wind velocity, and width of PV module. 
A PVT air system included a PVT air collector connected to a short length duct with fan for the air transfer had been designed by Bambrook [23]. In minimizing the energy output, the collector depth and the air mass flow rate had been optimized.

Yang and Athienitis [24] presented a prototype of open loop air-based building integrated PVT system with a single inlet. The study had involved through a wide-ranging series of experiments in a full scale solar simulator. A numerical control volume model was presented and confirmed based on the experimental results.

A study on basis of annual overall thermal energy and exergy for New Delhi has been carried out past five years by using energy metrics of PVT air collector. They have investigated that, by including the cost of energy in the manufacturing of components of the PVT module air collector, the payback and efficiency resulted vice versa pattern as the payback increased, the efficiency decreased respectively [25].

Then, the single and double-pass design of PVT had been improved for energy and exergy analysis which had been done by Kamthania et al. [26]. Yet, by all means, the superiority of double-pass PVT as the best design had been ratified as well from the outcome of this study.

Tonui and Tripanagnostopoulos [27] have developed an economical modification technique which in the meantime the heat transfer of PVT air collector can be improved. From the attained data of experiments, they had been validated for both glazed and unglazed PVT model as had been designated in the experimental procedure.

The performance of PVT was influenced by the thickness of the glass as well as the module's temperature and the inlet flow [28]. Substituting mono facial PV cells with bifacial PV cells has led to the development of bifacial PVT panels. The integration of an aluminum reflector contributes to $40 \%$ increment in both energy generation [29].

A study of four air-based bifacial PVT panels were designed, resulting maximum efficiencies of $45 \%$ to $63 \%$ were observed for the double-path-parallel bifacial PVT panel. It contributed as second preferred design due to its performance in generating up to $20 \%$ additional total energy compared to the single-path panel. However, for the analysis on daily average exergy efficiency, the double-path-parallel panel is $0.35 \%$ lower than that of a single-path panel [30].

A study which was configuring single and double glass were developed and analyzed. It had been observed that greater duct depth affect single glass more than double glass configuration. It had been preferred for the PVT system to be designated with effective economical calculations in order to ensure low life rate for affordable system [31].

\section{ENERGY AND EXERGY EFFICIENCY OF AIR-BASED PVT SYSTEMS}

An assessment of proposed design by Solanki et al. [32] on the performance of air- based PVT had been conducted. The experiment was carried out under variable insolation and air flow rate by constituting three mono crystalline silicon glass to tedlar type PV modules. They attained $42 \%, 8.4 \%$ and $50 \%$ respectively for the efficiencies of thermal, electrical and system.

An exergy analysis of flat plate air based PVT system had been conducted by Srimanickam et al. [33]. It had been attained that the system had generated $9.78 \%, 24.22 \%, 44.84 \%$ and $11.23 \%$ respectively of electrical, thermal, overall energy efficiency and exergy efficiency. Various energy and exergy efficiencies of air-based PVT systems as shown in Table 1.

Table 1. Performance of air-based PVT Systems

\begin{tabular}{|c|c|c|c|c|c|c|c|}
\hline \multirow[t]{2}{*}{ Year } & \multirow[t]{2}{*}{ Author(s) } & \multirow[t]{2}{*}{ Study } & \multirow{2}{*}{$\begin{array}{c}\text { Performance } \\
\text { Analyses }\end{array}$} & \multicolumn{3}{|c|}{ Energy Efficiencies (\%) } & \multirow{2}{*}{$\begin{array}{c}\text { PVT } \\
\text { Exergy } \\
\text { Efficiency } \\
(\%)\end{array}$} \\
\hline & & & & Thermal & PV & Overall & \\
\hline 1996 & $\begin{array}{l}\text { Sopian et } \\
\text { al. [19] }\end{array}$ & $\begin{array}{l}\text { Experimental and } \\
\text { theoretical }\end{array}$ & Energy analysis & 35 & 7.5 & 50 & NA \\
\hline 1997 & $\begin{array}{c}\text { Garg and } \\
\text { Adhikari } \\
\text { [31] }\end{array}$ & Experimental & Energy analysis & 42 & 8.4 & 50.4 & NA \\
\hline 2000 & $\begin{array}{l}\text { Hegazy } \\
{[16]}\end{array}$ & Experimental & Energy analysis & 58 & 8.1 & 55 & NA \\
\hline 2000 & $\begin{array}{l}\text { Sopian et } \\
\text { al. [34] }\end{array}$ & Experimental & Energy analysis & 60 & NA & NA & NA \\
\hline 2003 & $\begin{array}{c}\text { Saitoh et al. } \\
\text { [35] }\end{array}$ & $\begin{array}{l}\text { Experimental and } \\
\text { theoretical }\end{array}$ & Energy analysis & $40-50$ & $10-13$ & NA & NA \\
\hline 2005 & $\begin{array}{l}\text { Othman et } \\
\text { al. [36] }\end{array}$ & $\begin{array}{l}\text { Experimental and } \\
\text { theoretical }\end{array}$ & Energy analysis & NA & NA & $39-70$ & NA \\
\hline
\end{tabular}

Indonesian J Elec Eng \& Comp Sci, Vol. 14, No. 3, June 2019 : 1134 - 1140 


\begin{tabular}{|c|c|c|c|c|c|c|c|}
\hline 2006 & $\begin{array}{l}\text { Othman et } \\
\text { al. [37] }\end{array}$ & Experimental & Energy analysis & NA & 9 & $38-70$ & NA \\
\hline 2007 & $\begin{array}{c}\text { Tonui and } \\
\text { Tripanagno } \\
\text { stopoulos } \\
{[38]}\end{array}$ & Experimental & Energy analysis & 52 & $9-10$ & $61-62$ & NA \\
\hline 2007 & $\begin{array}{l}\text { Tiwari and } \\
\text { Sodha [39] }\end{array}$ & $\begin{array}{l}\text { Experimental and } \\
\text { theoretical }\end{array}$ & Energy analysis & NA & 10 & 40 & NA \\
\hline 2007 & $\begin{array}{c}\text { Alfegi et al. } \\
\text { [40] }\end{array}$ & Experimental & Energy analysis & $15.2-26.4$ & $11.4-12.7$ & $\begin{array}{l}26.6- \\
39.13\end{array}$ & NA \\
\hline 2008 & $\begin{array}{c}\text { Alfegi et al. } \\
\text { [41] }\end{array}$ & Experimental & Energy analysis & $17-26.43$ & $10.5-12.09$ & $27.5-40.4$ & NA \\
\hline 2008 & $\begin{array}{l}\text { Nayak and } \\
\text { Tiwari [42] }\end{array}$ & $\begin{array}{l}\text { Experimental and } \\
\text { theretical }\end{array}$ & $\begin{array}{l}\text { Energy-exergy } \\
\text { analysis }\end{array}$ & NA & NA & NA & 4 \\
\hline 2009 & $\begin{array}{c}\text { Joshi et al. } \\
\text { [43] }\end{array}$ & Experimental & Energy analysis & $26.4-30.5$ & $9.5-11$ & $41.6-47.4$ & NA \\
\hline 2009 & $\begin{array}{c}\text { Alfegi et al. } \\
\text { [44] }\end{array}$ & Experimental & Energy analysis & NA & NA & $49.1-62.8$ & NA \\
\hline 2009 & $\begin{array}{c}\text { Dubey et } \\
\text { al. [45] }\end{array}$ & $\begin{array}{l}\text { Experimental and } \\
\text { theoretical }\end{array}$ & Energy analysis & NA & NA & $\begin{array}{l}9.75- \\
10.41\end{array}$ & NA \\
\hline 2009 & $\begin{array}{l}\text { Ibrahim et } \\
\text { al. [46] }\end{array}$ & Theoretical & Energy analysis & $32.4-50.1$ & 11.9 & NA & NA \\
\hline 2010 & $\begin{array}{c}\text { Agrawal } \\
\text { and Tiwari } \\
\text { [47] }\end{array}$ & $\begin{array}{l}\text { Experimental and } \\
\text { theoretical }\end{array}$ & $\begin{array}{l}\text { Energy-exergy } \\
\text { analysis }\end{array}$ & NA & NA & 53.7 & NA \\
\hline 2010 & $\begin{array}{c}\text { Agrawal } \\
\text { and Tiwari } \\
{[48]}\end{array}$ & $\begin{array}{l}\text { Experimental and } \\
\text { theoretical }\end{array}$ & $\begin{array}{l}\text { Energy- } \\
\text { economic } \\
\text { analysis }\end{array}$ & 33.54 & 7.13 & NA & NA \\
\hline 2010 & $\begin{array}{l}\text { Sarhaddi et } \\
\text { al. [14] }\end{array}$ & $\begin{array}{l}\text { Experimental and } \\
\text { theoretical }\end{array}$ & Energy analysis & 17.18 & 10 & 45 & NA \\
\hline 2010 & $\begin{array}{l}\text { Sarhaddi et } \\
\text { al. [15] }\end{array}$ & $\begin{array}{l}\text { Experimental and } \\
\text { theoretical }\end{array}$ & $\begin{array}{l}\text { Energy-exergy } \\
\text { analysis }\end{array}$ & 17.18 & 10 & 45 & 10.75 \\
\hline 2010 & $\begin{array}{c}\text { Shahsavar } \\
\text { and Ameri } \\
\text { [49] }\end{array}$ & $\begin{array}{l}\text { Experimental and } \\
\text { theoretical }\end{array}$ & Energy analysis & 60 & 9.5 & 72 & NA \\
\hline 2011 & $\begin{array}{c}\text { Agrawal } \\
\text { and Tiwari } \\
{[50]}\end{array}$ & Theoretical & $\begin{array}{l}\text { Energy-exergy } \\
\text { analysis }\end{array}$ & 70.62 & NA & NA & NA \\
\hline 2012 & $\begin{array}{c}\text { Amori and } \\
\text { Al-Najjar } \\
{[51]}\end{array}$ & Theoretical & Energy analysis & $19.4-22.8$ & $9-12.3$ & $47.8-53.6$ & NA \\
\hline 2012 & $\begin{array}{l}\text { Agrawal et } \\
\text { al. [52] }\end{array}$ & $\begin{array}{l}\text { Experimental and } \\
\text { theoretical }\end{array}$ & $\begin{array}{c}\text { Energy-exergy } \\
\text { analysis }\end{array}$ & 35.7 & 12.4 & NA & NA \\
\hline 2013 & $\begin{array}{c}\text { Agrawal } \\
\text { and Tiwari } \\
{[53]}\end{array}$ & Experimental & $\begin{array}{l}\text { Energy-exergy- } \\
\text { environmental } \\
\text { analysis }\end{array}$ & 32 & NA & NA & NA \\
\hline 2014 & $\begin{array}{c}\text { Yang and } \\
\text { Athienitis } \\
{[24]}\end{array}$ & $\begin{array}{l}\text { Experimental and } \\
\text { theoretical }\end{array}$ & Energy analysis & 27.1 & 10 & NA & NA \\
\hline 2014 & Kim et al. & Experimental & Energy analysis & 22 & 15 & NA & NA \\
\hline 2014 & $\begin{array}{c}\text { Amori and } \\
\text { Al Raheem } \\
{[55]}\end{array}$ & Experimental & Energy analysis & $46-62$ & $8.3-10.4$ & NA & NA \\
\hline 2015 & $\begin{array}{l}\text { Li et al. } \\
\text { [56] }\end{array}$ & $\begin{array}{l}\text { Experimental and } \\
\text { theoretical }\end{array}$ & Energy analysis & 50 & $11.9-12.4$ & 77.7 & NA \\
\hline 2015 & $\begin{array}{l}\text { Good et al. } \\
\text { [57] }\end{array}$ & Experimental & Energy analysis & 71.5 & 17.4 & NA & NA \\
\hline 2015 & $\begin{array}{l}\text { Ahn et al. } \\
\text { [58] }\end{array}$ & Experimental & Energy analysis & 23 & 15 & 38 & NA \\
\hline 2015 & $\begin{array}{l}\text { Jahromi et } \\
\text { al. [59] }\end{array}$ & Theoretical & $\begin{array}{c}\text { Energy-exergy- } \\
\text { economic } \\
\text { analysis }\end{array}$ & $51.6-52$ & $7.5-8.7$ & NA & $9.6-9.7$ \\
\hline 2015 & $\begin{array}{c}\text { Kamel and } \\
\text { Fung [60] }\end{array}$ & Theoretical & Energy analysis & $32.8-41$ & $16-17$ & NA & NA \\
\hline 2015 & $\begin{array}{l}\text { Rajoria et } \\
\text { al. [61] }\end{array}$ & Theoretical & $\begin{array}{c}\text { Energy-exergy- } \\
\text { enviro- } \\
\text { economic } \\
\text { analysis }\end{array}$ & $12.1-28.1$ & $3.1-9.1$ & NA & NA \\
\hline 2016 & $\begin{array}{c}\text { Gholampou } \\
\mathrm{r} \text { and Ameri } \\
{[62]}\end{array}$ & Experimental & $\begin{array}{c}\text { Energy-exergy } \\
\text { analysis }\end{array}$ & 55 & NA & 69.91 & 8.66 \\
\hline 2016 & $\begin{array}{l}\text { Rounis et } \\
\text { al. [63] }\end{array}$ & Theoretical & Energy analysis & 48 & 16.5 & NA & NA \\
\hline
\end{tabular}




\begin{tabular}{|c|c|c|c|c|c|c|c|}
\hline 2016 & $\begin{array}{l}\text { Mojumder } \\
\text { et al. [64] }\end{array}$ & $\begin{array}{l}\text { Experimental and } \\
\text { theoretical }\end{array}$ & Energy analysis & 56.19 & 13.75 & NA & NA \\
\hline 2016 & $\begin{array}{c}\text { Hazami et } \\
\text { al. [65] }\end{array}$ & $\begin{array}{l}\text { Experimental and } \\
\text { theoretical }\end{array}$ & $\begin{array}{c}\text { Energy-exergy } \\
\text { analysis }\end{array}$ & 50 & 15 & NA & 14.8 \\
\hline 2016 & $\begin{array}{l}\text { Tiwari and } \\
\text { Tiwari [66] }\end{array}$ & Experimental & $\begin{array}{c}\text { Energy-exergo- } \\
\text { economic } \\
\text { analysis }\end{array}$ & NA & NA & 68.5 & NA \\
\hline 2016 & $\begin{array}{l}\text { Slimani et } \\
\text { al. [67] }\end{array}$ & $\begin{array}{l}\text { Experimental and } \\
\text { theoretical }\end{array}$ & Energy analysis & 70 & 10.5 & 90 & NA \\
\hline 2017 & $\begin{array}{c}\text { Salem et al. } \\
{[67]}\end{array}$ & Experimental & $\begin{array}{l}\text { Energy-exergy } \\
\text { analysis }\end{array}$ & $31.6-57.9$ & $17.7-38.4$ & $59.3-92$ & $11.1-13.5$ \\
\hline 2018 & $\begin{array}{l}\text { Fudholi et } \\
\text { al. [10] }\end{array}$ & $\begin{array}{l}\text { Experimental and } \\
\text { theoretical }\end{array}$ & $\begin{array}{l}\text { Energy-exergy } \\
\text { analysis }\end{array}$ & $21.3-82.9$ & $9.87-11.34$ & $\begin{array}{l}31.21- \\
94.24\end{array}$ & $\begin{array}{c}12.66- \\
12.91\end{array}$ \\
\hline
\end{tabular}

\section{CONCLUSIONS}

Air-based PVT systems had achieved great efficiencies in producing both thermal and electrical energy. Maximum efficiency value of thermal gain attained up to $82.9 \%$ while different study had achieved $38.4 \%$ of electrical energy. Latest exergy study presented exergy efficiency obtained more than $12 \%$. Further research and analysis would like to be recommended in order to obtain best system in generating useful energy, portrayed by the exergy value, can be achieve by the system.

\section{ACKNOWLEDGEMENT}

The authors gratefully acknowledge the funding from the USAID through SHERA program-Centre for Development of Sustainable Region (CDSR). The authors would like to thanks the UKM for support.

\section{REFERENCES}

[1] Nazri NS, Fudholi A, Bakhtyar B, Yen CH, Ibrahim A, Ruslan MH, Mat S, Sopian K. (2018), Energy economic analysis of photovoltaic-thermal-thermoelectric (PVT-TE) air collectors. Renewable and Sustainable Energy Review 92, 187-97.

[2] Nazri NS, Fudholi A, Ruslan MH, Sopian K. (2018), Mathematical modeling of photovoltaic thermalthermoelectric (PVT-TE) air collector. International Journal of Power Electronics and Drive Systems (IJPEDS), 9 (2), 795-802.

[3] Zohri M, Nurato N, Fudholi A (2017), Photovoltaic thermal (PVT) system with and without fins Collector: Theoretical Approach. International Journal of Power Electronics and Drive System (IJPEDS) 8(4), 1756-63.

[4] Fudholi A, Sopian K (2018), Review on exergy and energy analysis of solar air heater. International Journal of Power Electronics and Drive Systems (IJPEDS) 9 (1), 420-26.

[5] Fudholi A, Sopian K (2018), Review on solar collector for agricultural produce. International Journal of Power Electronics and Drive Systems (IJPEDS) 9 (1), 414-19.

[6] Fudholi A, Sopian K (2018), R\&D of Photovoltaic Thermal (PVT) Systems: an overview. International Journal of Power Electronics and Drive Systems (IJPEDS) 9 (2), 803-10.

[7] Fudholi A, Haw LC, Sopian K \& Abdulmula AMO (2018), Primary study of tracking photovoltaic system for mobile station in Malaysia. International Journal of Power Electronics and Drive Systems (IJPEDS) 9 (1), 427-32.

[8] Fudholi, A., Othman, M. Y., Ruslan, M. H., Yahya, M., Zaharim, A., \& Sopian, K. (2011). Design and testing of solar dryer for drying kinetics of seaweed in Malaysia. Recent Research in Geography, Geology, Energy, Environment and Biomedicine, 119-124.

[9] Aisyah CN, Fudholi A, Othman, MY, Ibrahim A, Ruslan MH, Sopian, K, (2018). Kecekapan pengumpul PV/T menggunakan pengumpul terma reka bentuk pilin (Efficiency of PV/T collector using spiral thermal absorber design), Sains Malaysiana 47 (4), 853-859.

[10] Fudholi A, Zohri M, Jin GL, Ibrahim A, Yen CH, Othman MY, Ruslan MH, Sopian K (2018), Energy and exergy analyses of pho-tovoltaic thermal collector with $\nabla$-groove. Solar Energy 159, 742-50.

[11] Razali NFM, Fudholi A, Ruslan MH, Sopian K, (2019), Improvement of photovoltaic module efficiency using nanofluids flow. Indonesian Journal of Electrical Engineering and Computer Science 13 (1), 324-330.

[12] Zohri M, Bakti LD, Fudholi A (2018), Exergy assessment of photovoltaic thermal with v-groove collector using theoretical study. TELKOMNIKA 16(2), 550-57.

[13] Razali NFM, Fudholi A, Ruslan MH, Sopian K, (2019). Review of water-nanofluid based photovoltaic/thermal (PV/T) systems. International Journal of Electrical and Computer Engineering (IJECE) 9 (1), 134-140.

[14] Sarhaddi, F., Farahat, S., Ajam, H., Behzadmehr, A., Mahdavi Adeli, M., (2010). An improved thermal and electrical model for a solar photovoltaic thermal (PV/T) air collector. Applied Energy 87, 2328-2339.

[15] Sarhaddi, F., Farahat, S., Ajam, H., Behzadmehr, A., (2010).Exergetic performance assessment of a solar photovoltaic thermal (PV/T) air collector. Energy and Buildings 42, 2184-2199. 
[16] Hegazy, A.A., (2000). Comparative study of the performances of four photovoltaic/thermal solar air collectors. Energy Conversion and Management 41, 861-881.

[17] Shan, F., Tang, F., Cao, L. and Fang, G., (2014), Comparative simulation analyses on dynamic performances of photovoltaic-thermal solar collectors with different configurations. Energy Conversion and Management, 87, pp.778-786.

[18] Khelifa, A., Touafek, K., Moussa, H. B., Tabet, I., \& Haloui, H. (2015). Analysis of a hybrid solar collector photovoltaic thermal (PVT). Energy Procedia, 74, 835-843.

[19] Sopian, K., Yigit, K. S., Liu, H. T., Kakac, S., \& Veziroglu, T. N. (1996). Performance analysis of photovoltaic thermal air heaters. Energy Conversion and Management, 37(11), 1657-1670.

[20] Naveed, A.T., Kang, E.C., Lee, E.J., (2006), "Effect of Unglazed Transpired Collector on the Performance of a Polycrystalline Silicon Photovoltaic Module,” Journal of Solar Energy Engineering, vol. 128, no. 3, p. 349.

[21] Arulanandam, S.J., Hollands, K.G.T., Brundrett, E., (1999), "A CFD heat transfer analysis of the transpired solar collector under no-wind conditions," Solar Energy, vol. 67, no. 1-3, pp. 93-100.

[22] S. Li and P. Karava, (2012), "Evaluation of turbulence models for airflow and heat transfer prediction in BIPV/T systems optimization," Energy Procedia, vol. 30, no. 30, pp. 1025-1034.

[23] S. Bambrook, (2011), "Investigation of photovoltaic thermal air systems to create a zero energy house in Sydney".

[24] T. Yang and A. K. Athienitis, (2014), "A study of design options for a building integrated photovoltaic/thermal (BIPV/T) system with glazed air collector and multiple inlets," Solar Energy, vol. 104, pp. 82-92.

[25] S. Agrawal and G. N. Tiwari, (2013), "Enviroeconomic analysis and energy matrices of glazed hybrid photovoltaic thermal module air collector," Solar Energy, vol. 92, pp. 139-146.

[26] Kamthania, D., Nayak, S., Tiwari, G.N., (2011), "Energy and exergy analysis of a hybrid photovoltaic thermal double pass air collector," Applied Solar Energy, vol. 47, no. 3, pp. 199-206.

[27] Tonui, J.K., Tripanagnostopoulos, Y., (2008), "Performance improvement of PV/T solar collectors with natural air flow operation," Solar Energy, vol. 82, no. 1, pp. 1-12.

[28] S. Agrawal and G. N. Tiwari, (2015), "Performance analysis in terms of carbon credit earned on annualized uniform cost of glazed hybrid photovoltaic thermal air collector," Solar Energy, vol. 115, pp. 329-340.

[29] Robles-Ocampo, B., Ruíz-Vasquez, E., Canseco-Sánchez, H., Cornejo-Meza, R.C., Trápaga-Martínez, G., GarcíaRodriguez, F.J., González-Hernández, J., Vorobiev, Y.V., (2007), "Photovoltaic/thermal solar hybrid system with bifacial PV module and transparent plane collector," Solar Energy Materials and Solar Cells, vol. 91, no. 20, pp. 1966-1971, 2007.

[30] [30] Ooshaksaraei, P., Sopian, K., Zulkifli, R., \& Zaidi, S. H. (2013). Characterization of air-based photovoltaic thermal panels with bifacial solar cells. International Journal of Photoenergy, 2013.

[31] H. P. Garg and R. S. Adhikari, (1997), "Conventional hybrid photovoltaic/thermal (PV/T) air heating collectors: steady-state simulation," Renewable Energy, vol. 11, no. 3, pp. 363-385.

[32] Solanki, S. C., Dubey, S., \& Tiwari, A. (2009). Indoor simulation and testing of photovoltaic thermal (PV/T) air collectors. Applied energy, 86(11), 2421-2428.

[33] Srimanickam, B., Vijayalakshmi, M.M., Natarajan, E., (2015), "Experimental Study of Exergy Analysis on Flat Plate Solar Photovoltaic Thermal (PV/T) Hybrid System," Applied Mechanics and Materials, vol. 787, pp. 82-87.

[34] Sopian, K., Liu, H.., Kakac, S., Veziroglu, T., (2000), Performance of a double pass photovoltaic thermal solar collector suitable for solar drying systems. Energy Conversion and Management.

[35] Saitoh, H., Hamada, Y., Kubota, H., Nakamura, M., Ochifuji, K., Yokoyama, S., Nagano, K., (2003), Field experiments and analyses on a hybrid solar collector. Applied Thermal Engineering 2003

[36] Othman, M. Y. H., Yatim, B., Sopian, K., \& Bakar, M. N. A. (2005). Performance analysis of a double-pass photovoltaic/thermal (PV/T) solar collector with CPC and fins. Renewable energy, 30(13).

[37] Othman, M. Y. H., Sopian, K., Yatim, B., \& Daud, W. R. W. (2006). Development of advanced solar assisted drying systems. Renewable Energy, 31(5), 703-709.

[38] J. K. Tonui and Y. Tripanagnostopoulos, (2007), "Air-cooled PV/T solar collectors with low cost performance improvements," Solar Energy, vol. 81, no. 4, pp. 498-511.

[39] A. Tiwari and M. S. Sodha, (2007), "Parametric study of various configurations of hybrid PV/thermal air collector: Experimental validation of theoretical model," Solar Energy Materials and Solar Cells, vol. 91, no. 1, pp. 17-28.

[40] Alfegi, E. M. A., Sopian, K., Othman, M. Y. H., \& Yatim, B. B. (2006). Transient mathematical model of both side single pass photovoltaic thermal air collector. $m h, 1000(0054), 0-1$.

[41] Alfegi, M. E. A., Sopian, K., Othman, M. Y. H., \& Yatim, B. B. (2008). Experimental investigation of single pass, double duct photovoltaic thermal (PV/T) air collector with CPC and fins. American Journal of applied sciences, 5(7), 866-871.

[42] S. Nayak and G. N. Tiwari, (2008), "Energy and exergy analysis of photovoltaic/thermal integrated with a solar greenhouse," Energy and Buildings, vol. 40, no. 11, pp. 2015-2021.

[43] Joshi, A. S., Tiwari, A., Tiwari, G. N., Dincer, I., \& Reddy, B. V. (2009). Performance evaluation of a hybrid photovoltaic thermal (PV/T) (glass-to-glass) system. International Journal of Thermal Sciences, 48(1), 154-164.

[44] E. M. A. Alfegi et al, (2009), The effects of flow rates on the performance of finned single pass, double duct photovoltaic thermal solar air heaters. European journal of scientific research., vol. 25, no. 2. EuroJournals, 2009.

[45] Dubey, S., Sandhu, G. S., \& Tiwari, G. N. (2009). Analytical expression for electrical efficiency of PV/T hybrid air collector. Applied Energy, 86(5), 697-705.

[46] Ibrahim, A., Othman, M. Y., Ruslan, M. H., Alghoul, M., Yahya, M., Zaharim, A., \& Sopian, K. (2009). Performance of photovoltaic thermal collector (PVT) with different absorbers design. WSEAS Transactions on Environment and Development, 5(3), 321-330. 
[47] Agrawal, B., \& Tiwari, G. N. (2010). Optimizing the energy and exergy of building integrated photovoltaic thermal (BIPVT) systems under cold climatic conditions. Applied Energy, 87(2), 417-426.

[48] Agrawal, B., \& Tiwari, G. N. (2010). Life cycle cost assessment of building integrated photovoltaic thermal (BIPVT) systems. Energy and Buildings, 42(9), 1472-1481.

[49] Shahsavar, A., \& Ameri, M. (2010). Experimental investigation and modeling of a direct-coupled PV/T air collector. Solar Energy, 84(11), 1938-1958.

[50] Agrawal, S., \& Tiwari, G. N. (2011). Energy and exergy analysis of hybrid micro-channel photovoltaic thermal module. Solar Energy, 85(2), 356-370.

[51] Amori, K. E., \& Al-Najjar, H. M. T. (2012). Analysis of thermal and electrical performance of a hybrid (PV/T) air based solar collector for Iraq. Applied Energy, 98, 384-395.

[52] Agrawal, S., Tiwari, G. N., \& Pandey, H. D. (2012). Indoor experimental analysis of glazed hybrid photovoltaic thermal tiles air collector connected in series. Energy and buildings, 53, 145-151.

[53] Agrawal, S., \& Tiwari, G. N. (2013). Overall energy, exergy and carbon credit analysis by different type of hybrid photovoltaic thermal air collectors. Energy conversion and Management, 65, 628-636.

[54] Kim, J. H., Park, S. H., \& Kim, J. T. (2014). Experimental performance of a photovoltaic-thermal air collector. Energy Procedia, 48, 888-894.

[55] Amori, K. E., \& Abd-AlRaheem, M. A. (2014). Field study of various air based photovoltaic/thermal hybrid solar collectors. Renewable Energy, 63, 402-414.

[56] Li, Y., Zhang, G., Lv, G. Z., Zhang, A. N., \& Wang, R. Z. (2015). Performance study of a solar photovoltaic air conditioner in the hot summer and cold winter zone. Solar Energy, 117, 167-179.

[57] Good, C., Andresen, I., \& Hestnes, A. G. (2015). Solar energy for net zero energy buildings-A comparison between solar thermal, PV and photovoltaic-thermal (PV/T) systems. Solar Energy, 122, 986-996.

[58] Ahn, J. G., Kim, J. H., \& Kim, J. T. (2015). A study on experimental performance of air-type PV/T collector with HRV. Energy Procedia, 78, 3007-3012.

[59] Jahromi, S. N., Vadiee, A., \& Yaghoubi, M. (2015). Exergy and economic evaluation of a commercially available $\mathrm{PV} / \mathrm{T}$ collector for different climates in Iran. Energy Procedia, 75, 444-456.

[60] Kamel, R. S., \& Fung, A. S. (2015). Modelling and Characterization of Transparent Building Integrated PV/T Collector. Energy Procedia, 78, 1871-1876.

[61] Rajoria, C. S., Agrawal, S., Tiwari, G. N., \& Chaursia, G. S. (2015). Exergetic and enviroeconomic analysis of semitransparent PVT array based on optimum air flow configuration and its comparative study. Solar Energy, 122, 1138-1145.

[62] M. Gholampour and M. Ameri, (2016), "Energy and exergy analyses of Photovoltaic/Thermal flat transpired collectors: Experimental and theoretical study," Applied Energy, vol. 164, pp. 837-856,.

[63] Rounis, E. D., Athienitis, A. K., \& Stathopoulos, T. (2016). Multiple-inlet Building Integrated Photovoltaic/Thermal system modelling under varying wind and temperature conditions. Solar Energy, 139, 157-170.

[64] Mojumder, J. C., Chong, W. T., Ong, H. C., \& Leong, K. Y. (2016). An experimental investigation on performance analysis of air type photovoltaic thermal collector system integrated with cooling fins design. Energy and Buildings, 130, 272-285.

[65] Hazami, M., Riahi, A., Mehdaoui, F., Nouicer, O., \& Farhat, A. (2016). Energetic and exergetic performances analysis of a PV/T (photovoltaic thermal) solar system tested and simulated under to Tunisian (North Africa) climatic conditions. Energy, 107, 78-94.

[66] S. Tiwari and G. N. Tiwari, (2016), "Exergoeconomic analysis of photovoltaic-thermal (PVT) mixed mode greenhouse solar dryer," Energy, vol. 114, pp. 155-164.

[67] Slimani, M. E. A., Amirat, M., Bahria, S., Kurucz, I., \& Sellami, R. (2016). Study and modeling of energy performance of a hybrid photovoltaic/thermal solar collector: Configuration suitable for an indirect solar dryer. Energy conversion and management, 125, 209-221.

[68] Salem, M. R., Ali, R. K., \& Elshazly, K. M. (2017). Experimental investigation of the performance of a hybrid photovoltaic/thermal solar system using aluminium cooling plate with straight and helical channels. Solar Energy, 157, 147-156. 